\title{
Degeneration of Phrenic Motor Neurons Induces Long-Term Diaphragm Deficits following Mid-Cervical Spinal Contusion in Mice
}

\author{
Charles Nicaise,,2 Rajarshi Putatunda,, Tamara J. Hala,, Kathleen A. Regan,, David M. Frank, \\ Jean-Pierre Brion, ${ }^{2}$ Karelle Leroy, ${ }^{2}$ Roland Pochet, ${ }^{2}$ Megan C. Wright, ${ }^{3}$ and Angelo C. Lepore ${ }^{1}$
}

\begin{abstract}
A primary cause of morbidity and mortality following cervical spinal cord injury (SCI) is respiratory compromise, regardless of the level of trauma. In particular, SCI at mid-cervical regions targets degeneration of both descending bulbospinal respiratory axons and cell bodies of phrenic motor neurons, resulting in deficits in the function of the diaphragm, the primary muscle of inspiration. Contusion-type trauma to the cervical spinal cord is one of the most common forms of human SCI; however, few studies have evaluated mid-cervical contusion in animal models or characterized consequent histopathological and functional effects of degeneration of phrenic motor neuron-diaphragm circuitry. We have generated a mouse model of cervical contusion SCI that unilaterally targets both C4 and C5 levels, the location of the phrenic motor neuron pool, and have examined histological and functional outcomes for up to 6 weeks post-injury. We report that phrenic motor neuron loss in cervical spinal cord, phrenic nerve axonal degeneration, and denervation at diaphragm neuromuscular junctions (NMJ) resulted in compromised ipsilateral diaphragm function, as demonstrated by persistent reduction in diaphragm compound muscle action potential amplitudes following phrenic nerve stimulation and abnormalities in spontaneous diaphragm electromyography (EMG) recordings. This injury paradigm is reproducible, does not require ventilatory assistance, and provides proof-of-principle that generation of unilateral cervical contusion is a feasible strategy for modeling diaphragmatic/respiratory deficits in mice. This study and its accompanying analyses pave the way for using transgenic mouse technology to explore the function of specific genes in the pathophysiology of phrenic motor neuron degeneration and respiratory dysfunction following cervical SCI.
\end{abstract}

Key words: cervical; contusion; mice; phrenic motor neuron; SCI

\section{Introduction}

$\mathbf{T}$ HE CERVICAL SPINAL CORD is affected in a large proportion of human spinal cord trauma cases. These spinal cord injuries (SCI) are often the result of road accidents, falls, sport injuries, or recreational activities, and occur most commonly in young individuals. Severe mid-cervical SCI leads to debilitating respiratory paralysis and often life-threatening emergencies, because of damage to the phrenic motor neuron pool that controls contraction of the diaphragm, the primary muscle of inspiration. ${ }^{1}$ Following cervical spinal cord trauma, deficits in breathing are attributed to disruption of supraspinal axons originating in bulbar primary respiratory centers, direct damage to motor neurons of the phrenic nucleus, and/or interruption of efferent phrenic motor nerve projections to the diaphragm. Given that the phrenic motor neuron- diaphragm circuit is directly affected by a large proportion of SCI cases, development and characterization of relevant cervical SCI models are crucial both for understanding the mechanisms of phrenic motor neuron loss following SCI and for developing therapies targeting protection and plasticity of this key motor neuron population. ${ }^{2}$

Contusion-type SCI models are some of the most clinically relevant experimental forms of spinal cord trauma. To date, the vast majority of spinal contusion models in the mouse have targeted thoracic levels, ${ }^{3-5}$ and it was only very recently that the first mouse model of bilateral cervical contusion SCI was described by Aguilar and Steward. ${ }^{6}$ Other available animal models of SCI include crushtype lesions, ${ }^{7}$ medullar compressions, ${ }^{8}$ and surgical tract axotomies. ${ }^{9-13}$ In particular, hemisection models at a high cervical level (C2) have been useful for studying the plasticity, recovery,

\footnotetext{
${ }^{1}$ Department of Neuroscience, Farber Institute for Neurosciences, Thomas Jefferson University Medical College, Philadelphia, Pennsylvania.

${ }^{2}$ Laboratory of General Histology, Neuroanatomy and Neuropathology, Université Libre de Bruxelles, Bruxelles, Belgium.

${ }^{3}$ Department of Biology, Arcadia University, Glenside, Pennsylvania.
} 
Table 1. Experimental Design

\section{Analyses}

1. CMAP recordings

2. Spontaneous EMG recordings

\begin{tabular}{lccc} 
Groups & 2 weeks & 6 weeks & Total \\
\hline Laminectomy & - & 10 & 10 \\
C4 + C5 double contusion & $\frac{8}{8}$ & $\frac{10}{20}$ & $\frac{18}{28}$
\end{tabular}

Analyses

1. Cresyl violet: lesion size and motor neuron counts

2. СT $\beta$ retrograde labeling and counts

3. Phrenic nerve histology

4. Diaphragm histology: thickness and myofiber size

Groups

Laminectomy

$\mathrm{C} 4+\mathrm{C} 5$ double contusion

$\begin{array}{rrr}- & 7 & 7 \\ \frac{5}{5} & \frac{7}{4} & \frac{12}{19}\end{array}$

Analyses

1. NMJ analysis

Groups

Laminectomy

$\mathrm{C} 4+\mathrm{C} 5$ double contusion

$\begin{array}{lll}- & 3 & 3 \\ \frac{3}{3} & \frac{3}{6} & \frac{6}{9}\end{array}$

All 28 animals were used for CMAP and EMG recordings. At the time of euthaniasia, animals were split into one of two groups. The first group (19 animals in total) was used for: 1) cresyl violet staining to quantify lesion size and to count total motor neuron numbers, 2) $\mathrm{CT} \beta^{+}$phrenic motor neuron counts, 3) phrenic nerve histology, and 4) diaphragm histology to quantify overall muscle thickness and individual myofiber size. The second group ( 9 animals in total) was used for diaphragm NMJ analysis by whole-mount immunohistochemistry.

CMAPs, compound muscle action potentials; $\mathrm{CT} \beta$, cholera toxin $\beta$; NMJ, neuromuscular junction; EMG, electromyography.

and repair of descending innervation of the phrenic nucleus, and notably were used to characterize the crossed-phrenic pathway in both mice ${ }^{13}$ and rats. ${ }^{14}$

Rats have traditionally been the preferred species used in the SCI research community for conducting most types of studies. They provide relative ease for conducting neurobehavioral assays, and their large size allows for collection of greater amounts of tissue for biochemical analysis and provides practical advantages when conducting invasive therapeutic interventions (e.g., gene and cell delivery). In addition, the formation of a cystic cavity in the rat spinal cord following contusion is in many ways similar to the human SCI lesion. ${ }^{15,16}$ Nevertheless, the development and widespread use of genetically modified animals, which are primarily in murine models, have paved the way for the manipulation of genes possibly relevant to the pathophysiology and treatment of SCI. ${ }^{4,5,11,12,17}$

The first published study characterizing cervical (C5 level) contusion SCI in the mouse focused on assessment of forelimb motor function following several graded bilateral injuries at 30, 75, or $100 \mathrm{kD}$ of impact force. ${ }^{6}$ Using grip strength testing, a graded deficit was observed in forelimb grip force that was proportional to impact severity. The authors also examined lesion size and loss of total cervical motor neurons, the majority of which innervate muscles of the forelimb. However, the effects specifically on phrenic motor neuron survival, innervation of the diaphragm, and functional respiratory outcomes, were not assessed.
Given the predominance of human SCI cases that affect midcervical spinal cord regions, as well as the high incidence of respiratory compromise in these patients, ${ }^{1}$ there is a high priority for investigating mechanisms of loss, protection and restoration of breathing. Therefore, we have in the present study addressed histopathological and functional changes in phrenic motor neuron-diaphragm circuitry in a mouse model of mid-cervical contusion SCI. We have specifically examined effects on phrenic motor neuron and phrenic nerve axonal loss, diaphragm muscle changes, and morphological and functional innervation of the diaphragm. In an attempt to affect a large proportion of the phrenic motor neuron pool, we targeted both $\mathrm{C} 4$ and C5 levels, as the phrenic nucleus is located approximately between levels C3 and $\mathrm{C} 5$ in mice. ${ }^{18}$ In order to study the long-term evolution of functional innervation of the diaphragm following injury, we characterized functional diaphragmatic changes out to 6 weeks post-injury. In addition, we histologically analyzed phrenic nerve-diaphragm circuitry at both 2 and 6 weeks post-injury in order to examine changes occurring between a relatively early and a more chronic time point, respectively. We have demonstrated that this unilateral cervical injury paradigm is reproducible with no need for breathing assistance and is accompanied by phrenic motor neuron loss; phrenic nerve axon degeneration, denervation, and subsequent partial reinnervation at the diaphragm NMJ; and reduction of phrenic nerve compound muscle action potentials (CMAP). These findings demonstrate a significant and persistent impairment in phrenic nucleus-phrenic nerve-diaphragm circuitry following contusion-type, mid-cervical SCI. This injury paradigm and accompanying analyses provide important tools for understanding mechanisms of phrenic motor neuron and diaphragm pathology following SCI. In addition, this model allows for the use of genetically modified animals for exploring the function of specific genes in the pathophysiology of respiratory motor neuron degeneration and functional respiratory compromise following cervical SCI. ${ }^{19-23}$

\section{Methods}

\section{Animals}

Eight to twelve-week-old male C57Bl/6 mice were obtained from Jackson Laboratory (Bar Harbor, ME) and housed in a controlled (light and temperature) environment with ad libitum access to food and water in the animal facility at Thomas Jefferson University. All care and treatment were conducted in compliance with the European Communities Council Directive (2010/63/EU, 86/ 609/EEC and 87-848/EEC), the National Institutes of Health (NIH) Guide for the Care and Use of Laboratory Animals, and the Thomas Jefferson University Institutional Animal Care and Use Committee (IACUC). Twenty-eight animals included in the study were divided into two groups: uninjured laminectomy-only controls and double SCI at both C4 and C5 levels. Table 1 summarizes the numbers of animals used for each procedure.

\section{Unilateral cervical spinal cord contusion}

Mice were anesthetized with a cocktail of ketamine $(120 \mathrm{mg} / \mathrm{kg})$ and xylazine $(5 \mathrm{mg} / \mathrm{kg})$. The dorsal skin and underlying muscle layers were incised along the midline between the spinous processes of $\mathrm{C} 2$ and $\mathrm{T} 1$ to expose the cervical region of the spinal cord. $^{24}$ The dorsal muscle layers were retracted, and the paravertebral muscles overlying C4-C6 were removed. Following unilateral laminectomy on the right side at $\mathrm{C} 4$ and $\mathrm{C} 5$ levels, mice were subjected to a double spinal contusion injury at both $\mathrm{C} 4$ and C5 levels using the Infinite Horizon (IH) impactor (Precision 
TABle 2. Infinite Horizon Impactor Parameters

\begin{tabular}{lccc}
\hline & Force $(\mathrm{kD})$ & Displacement $(\mu \mathrm{m})$ & Velocity $(\mathrm{mm} / \mathrm{s})$ \\
\hline C4 impact & $55.3 \pm 1.6$ & $640.4 \pm 44.6$ & $121.1 \pm 0.6$ \\
C5 impact & $53.6 \pm 1.6$ & $650.0 \pm 43.9$ & $123.0 \pm 0.6$ \\
\hline
\end{tabular}

Systems and Instrumentation, Lexington, KY). The full procedure included unilateral laminectomy on the right side at both levels $\mathrm{C} 4$ and $\mathrm{C} 5$, the extension of the laminectomy between the midline blood vessel and the lateral edge of the bone, and clamping of the spinous processes of $\mathrm{C} 2$ and $\mathrm{T} 2$ using toothed Adson forceps to stabilize the whole spinal column. The $1 \mathrm{~mm}$ impactor tip was then centered at each level before the impact and then raised $1.25 \mathrm{~mm}$ above the dura prior to impact. The medial edge of the impactor tip was positioned as close to the midline blood vessel as possible without actually overlapping it. This allowed the full diameter of the tip to fit without touching the lateral edge of the laminectomy. The C4 impact was first generated, followed by the impact at C5, at a force of $50 \mathrm{kD}$. The resulting impact force was collectively $54.4 \pm 1.1 \mathrm{kD}$ (mean \pm SEM) for all injuries. The parameters (i.e., displacement and velocity of the tip) recorded by the IH device after all impacts are presented in Table 2. Sham animals underwent the same surgery, including laminectomy, but did not receive contusion injury. Upon completion of the injury procedure, overlying muscles were closed in layers with sterile $4-0$ silk suture, and the skin incision was closed using sterile wound clips. After surgical procedures, animals were allowed to recover on a circulating warm water heating pad until awake and then returned to their home cages. Animals were monitored on a daily basis for 5 days, and measures were taken to avoid dehydration and to minimize any potential pain (subcutaneous administration of buprenorphine: $0.1 \mathrm{mg} / \mathrm{kg}$ ). Signs of bladder or bowel dysfunction were monitored on a daily basis following injury. For all animals, there was never any need for manual bladder expression and there were never any signs of blood in the urine. Discoloration or presence of blood in the animals' stool was also never observed.

\section{Retrograde anatomical tracing using fluorescent cholera toxin $\beta$ subunit (CT $\beta)$}

This procedure allows for specific visualization of the phrenic motor neuron pool via retrograde transport of fluorescent tracer from the ipsilateral hemidiaphragm. Two weeks before euthanasia, CT $\beta$ conjugated to Alexa555 (Life Technologies, Grand Island, NY), a retrograde axonal tracer, was injected unilaterally into the intrapleural space of the ipsilateral hemidiaphragm muscle. This method was modified from the original procedure described in rats by Mantilla et al. ${ }^{25}$ Following laparotomy under anesthesia, $5 \mu \mathrm{L}$ of CT $\beta(0.2 \%$ solution in distilled water $)$ were intrapleurally delivered through the diaphragm (2 injection sites of $2.5 \mu \mathrm{L}$ each) using a $10 \mu \mathrm{L}$ Hamilton syringe with an attached 33 gauge needle. To close the laparotomy, abdominal muscles were sutured with 4-0 suture, and skin was closed with wound clips. No complication (i.e. bleeding or pneumothorax) was ever observed following this procedure.

\section{Neurophysiology}

CMAP recordings. All neurophysiological recordings (CMAPs and electromyography [EMG]) were terminal procedures. Animals were anesthetized at either 2 weeks or 6 weeks post-injury in the same manner as described previously. With the animals under anesthesia, phrenic nerve conduction studies were performed with single stimulation ( $0.5 \mathrm{~ms}$ duration; $4 \mathrm{mV}$ amplitude $)$ at the neck via near nerve needle electrodes placed along the phrenic nerve. The ground needle electrode was placed in the tail, and the reference electrode was placed subcutaneously in the right abdominal region. Recording was obtained via a surface strip along the costal margin of the diaphragm, and CMAP amplitude was measured baseline to peak. Recordings were made using an ADI Powerlab 8/30 stimulator and BioAMP amplifier (ADInstruments, Colorado Springs, CO), followed by computer assisted data analysis (Scope 3.5.6, ADInstruments). A minimum of 10 tracings were taken following stimulation of the right phrenic nerve, and the average CMAP responses were recorded to ensure reproducibility.

Spontaneous EMG recordings. After CMAP recording and prior to being euthanized, animals received a laparotomy. Bipolar electrodes spaced by $3 \mathrm{~mm}$ were then inserted into several locations in the right hemidiaphragm. Activity was recorded and averaged during spontaneous breathing. The EMG signal was amplified, filtered through a band-pass filter $(50-3,000 \mathrm{~Hz})$, and analyzed using LabChart 7 software (ADInstruments). Parameters such as inspiratory burst per minute, discharge duration, and peak amplitude were averaged over $30 \mathrm{sec}$ sample periods.

\section{Histological analyses}

Tissue processing. Animals were anesthetized for euthanasia at either 2 weeks or 6 weeks post-injury in the same manner as described previously. Diaphragm muscle and phrenic nerves were freshly removed and further processed for histology or immunohistochemistry. Animals were transcardially perfused with $0.9 \%$ saline, followed by $4 \%$ paraformaldehyde infusion. Spinal cords were harvested, followed by preparation of cervical spinal cord segments by: 1) post-fixation step in paraformaldehyde overnight, 2) washing in $0.1 \mathrm{M}$ phosphate buffer, 3) cryoprotection in $30 \%$ sucrose $/ 0.1 \mathrm{M}$ phosphate buffer at $4{ }^{\circ} \mathrm{C}$ for 3 days, and 4) embedding in freezing medium. Spinal cord tissue blocks were cut in the sagittal or transverse planes at $30 \mu \mathrm{m}$ thickness. Sections were collected on glass slides and stored at $-20^{\circ} \mathrm{C}$ until analysis.

Lesion histology and motor neuron counting. Using every fifth slide, spinal cord sections were thawed, allowed to dry for $1 \mathrm{~h}$ at room temperature, stained with $0.5 \%$ cresyl violet acetate, and imaged using a Zeiss Imager M2 upright microscope. Using ImageJ software, the lesion area was outlined and quantified (investigator C.N.). Specifically, the lesion area was determined every $150 \mu \mathrm{m}$ on cresyl violet sections by tracing both the total area of the hemispinal cord ipsilateral to the lesion and the actual injury area to obtain two-dimensional values. Injury was defined as areas including both lost tissue and surrounding damaged tissue in which the normal anatomical structure of the spinal cord was lost. The lesion epicenter was defined as the section with the largest percent of lesioned tissue (relative to total hemi-cord area in the same section). Lesion volume was determined using the Cavalieri estimator of volume

$$
\mathrm{V}=\left[\Sigma\left(\mathrm{A}_{1}+\mathrm{A}_{2}+\ldots \mathrm{A}_{\mathrm{n}}\right) \times \mathrm{D}\right]-\left[\mathrm{A}_{\max } \times \mathrm{Y}\right]
$$

where $\mathrm{A}$ is the area of the lesion, D is the distance between measurements $(150 \mu \mathrm{m})$ and $\mathrm{Y}$ is the thickness $(30 \mu \mathrm{m})$ of each section).

To quantify myelin loss, every fifth slide was stained with Eriochrome R dye, according to a standard protocol. The percentage of areas with myelin loss at various distances from the lesion epicenter was calculated as the ratio of total area of unspared myelin per section to total ipsilateral hemi-cord area (investigator K.R.).

In cresyl violet stained sections, the total number of cervical motor neurons was quantified in a blind manner (investigator R.P.). Ventral horn was defined as gray matter ventral to the central canal. Only motor neurons with a clearly identifiable nucleus and a cell soma $>200 \mu \mathrm{m}^{2}$ were counted. 
To specifically count the number of phrenic motor neurons labeled with CT $\beta$ (via retrograde transport from the ipsilateral hemidiaphragm), transverse spinal cord sections (using every fifth slide) were thawed, allowed to dry for $1 \mathrm{~h}$ at room temperature, washed twice in $0.1 \mathrm{M}$ Tris- buffered saline (TBS), dipped into distilled water, and cover-slipped with fluorescence-compatible antifade reagent (ProLong Gold, Life Technologies, Grand Island, $\mathrm{NY}$ ). Across the entire cervical region, $\mathrm{CT} \beta^{+}$motor neurons were counted every fifth section in a blind manner (investigator C.N.), and an estimation of the total number of labeled cells in the ventral horn was calculated by multiplying by the number of counted $\mathrm{CT} \beta^{+}$cells by 5 . In addition, the number of $\mathrm{CT} \beta^{+}$cells was plotted at specific distances relative to the lesion epicenter.

Phrenic nerve histology. Upon removal, phrenic nerves were fixed in $4 \%$ glutaraldehyde solution for $1.5 \mathrm{~h}$, thoroughly washed in Millonig's buffer with $0.5 \%$ (w/v) glucose for $24 \mathrm{~h}$, postfixed in $2 \%(\mathrm{w} / \mathrm{v}) \mathrm{OsO}_{4}$ for $30 \mathrm{~min}$, dehydrated, and embedded in Epoxy resin LX112. Semithin cross sections (1-2 $\mu$ m thickness) were obtained and stained with $1 \%$ Toluidine blue (Sigma-Aldrich, St. Louis, MO) diluted in $2 \%$ sodium borate solution. Axonal density (i.e., number of axons divided by total nerve area) and their cross-sectional areas were counted in a blind manner (investigator K.L.) using ImageJ software, as previously reported. ${ }^{26}$ For electron microscopy analysis, ultrathin sections were cut using a diamond blade on an ultramicrotome. Ultrathin sections collected on nickel grids were counterstained with $2 \%$ uranyl acetate (dissolved in $50 \%$ ethanol) and Reynold's lead citrate solution and then observed with a Zeiss 109 electron microscope at $80 \mathrm{keV}$.

Diaphragm thickness analysis. Fresh hemidiaphragm was placed in tissue freezing medium (Triangle Biomedical Sciences, Durham, NC) and snap frozen in dimethylbutane cooled by liquid nitrogen. Frozen $10 \mu \mathrm{m}$ transverse sections were cut with a cryostat and stained with hematoxylin and eosin (EMD Chemicals, Gibbstown, NJ), according to standard protocols. Multiple transverse sections were obtained throughout the medial-lateral axis of the hemidiaphragm ipsilateral to the injury site for histological analysis. For quantification, three sections along the medial-lateral axis (spaced apart at $1 \mathrm{~mm}$ intervals) were analyzed per hemidiaphragm. On each section, the average of five thickness points equally distributed along the ventro-dorsal axis was measured in a blind manner (investigator D.F.) using ImageJ software. In total, 15 different locations were sampled along the entire hemidiaphragm for each animal. Myofiber size was also measured along the entire hemidiaphragm by averaging the area of a minimum of 100 cells.

NMJ analysis. Fresh hemidiaphragm muscle was dissected from each animal for whole-mount immunohistochemistry. ${ }^{29}$ Muscle was stretched, pinned down to Sylgard medium (Fisher Scientific, Pittsburgh, PA), and extensively cleaned to remove any connective tissue to allow for antibody penetration. Motor axons and their terminals were labeled with SMI-312R (Covance, Princeton, NJ) and SV2-s (DSHB, Iowa City, IA), respectively, and both antibody labelings were detected with FITC anti-mouse IgG, Fc $\gamma 1$ secondary antibody (Jackson ImmunoResearch Laboratories, West Grove, PA). Post-synaptic acetylcholine receptors (AChR) were labeled with rhodamine-conjugated alpha-bungarotoxin (Life Technologies). Labeled muscles were visualized (investigator M.W.) and quantified using an Axio Imager M2 (Zeiss, Thornwood, NY). Each junction was categorized as 1) normally innervated by the original axon, 2) completely denervated (no occupation of AChRs by nerve terminal), 3) partially denervated (partial occupation of AChRs by nerve terminal), 4) thinner diameter preterminal axons, and 5) multiply innervated NMJs. Both of these final categories are characteristic of recently reinnervated NMJs by regenerating axons (Wright and Son, 2007). For each muscle examined, a minimum of 100 NMJs were characterized. High-resolution confocal images were obtained for published images using a FluoView FV1000 confocal microscope (Olympus, Center Valley, PA).

\section{Statistical analysis}

Results were expressed as means \pm standard error of the mean (SEM). Statistical significance was assessed by Mann-Whitney test for comparisons involving two groups or by analysis of variance (one-way ANOVA) followed by multiple comparisons posthoc test (Bonferroni's method) for multiple comparisons. Statistics were computed with GraphPad Prism 5 (GraphPad Software, Inc., La Jolla, CA) and Sigma Plot 12 (Systat Software Inc., San Jose, CA) softwares. $p<0.05$ was considered as statistically significant.

\section{Results}

\section{Animal survival and neurological status following C4+C5 injury}

In order to target the injury to a large portion of the ipsilateral phrenic nucleus, mice received a double $50 \mathrm{kD}$ contusion at both $\mathrm{C} 4$ and C5 levels. This strategy was employed based on the knowledge that the majority of the phrenic motor neuron population is located between C3 and C6 levels in mice. ${ }^{18}$ Retrograde labeling of phrenic motor neurons from the ipsilateral hemidiaphragm confirmed that the greatest density of phrenic motor neurons was found at the $\mathrm{C} 4$ level. Upon completion of the double $\mathrm{C} 4+\mathrm{C} 5$ injury procedure, $70.4 \%$ of mice survived without the need for ventilatory assistance. All animals exhibited qualitatively ipsilateral forelimb paresis upon waking, and significant decrease of forelimb grip strength up to 6 weeks post-injury (data not shown).

\section{Decreased CMAP amplitude in ipsilateral hemi-diaphragm}

To electrophysiologically investigate whether contusion injury at C4 and C5 levels functionally impairs phrenic nerve-diaphragm circuitry, diaphragm CMAP amplitudes following phrenic nerve stimulation (nerve conduction studies) were obtained from surface recordings of the ipsilateral hemidiaphragm of both injured and laminectomy-only mice at 2 and 6 weeks post-injury. CMAP amplitudes in uninjured mice reached almost $4 \mathrm{mV}$ (Fig. 1A). Quantitatively, we observed at 2 weeks post-injury a significant decrease in CMAP amplitude in injured animals compared with the laminectomy group $(2.0 \pm 0.4 \mathrm{mV}$ vs. $3.8 \pm 0.3 \mathrm{mV}$ respectively, $p<0.01$ ) (Fig. 1B). By 6 weeks post-injury, CMAP amplitudes were similarly reduced in the injured group $(2.0 \pm 0.3 \mathrm{mV}, p<0.01)$ (Fig. 1C). Stimulation of the contralateral phrenic nerve did not result in any detectable CMAP response in the hemidiaphragm ipsilateral to the injury site (data not shown).

Needle EMG recordings from the ipsilateral hemidiaphragm did not demonstrate significant qualitative changes with respect to EMG amplitude at 2 weeks (Fig. 1E) and 6 weeks (Fig. 1F) after injury compared to the laminectomy-only group (Fig. 1D). For all conditions, motor unit amplitudes were in the $500-1,000 \mu \mathrm{V}$ range. However, a reduction in the burst inspiratory rate was observed over time following injury. These changes reached significance at 6 weeks post-injury ( $198 \pm 3$ vs. $123 \pm 17$ bursts per minute, $p<0.05$, in laminectomy and $\mathrm{C} 4+\mathrm{C} 5$ injury, respectively) (Fig. 1G). In addition, discharge duration in the ipsilesioned hemidiaphragm was significantly increased in injured animals compared with the laminectomy control group (130 \pm 12 vs. 
A

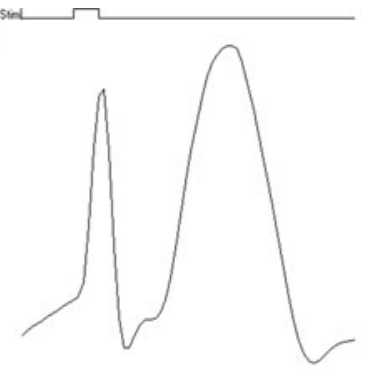

B

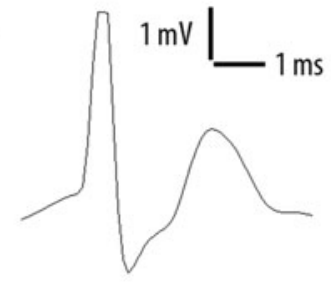

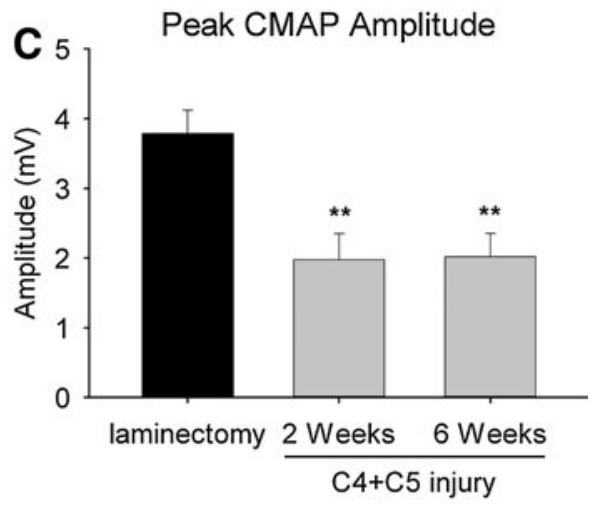

D
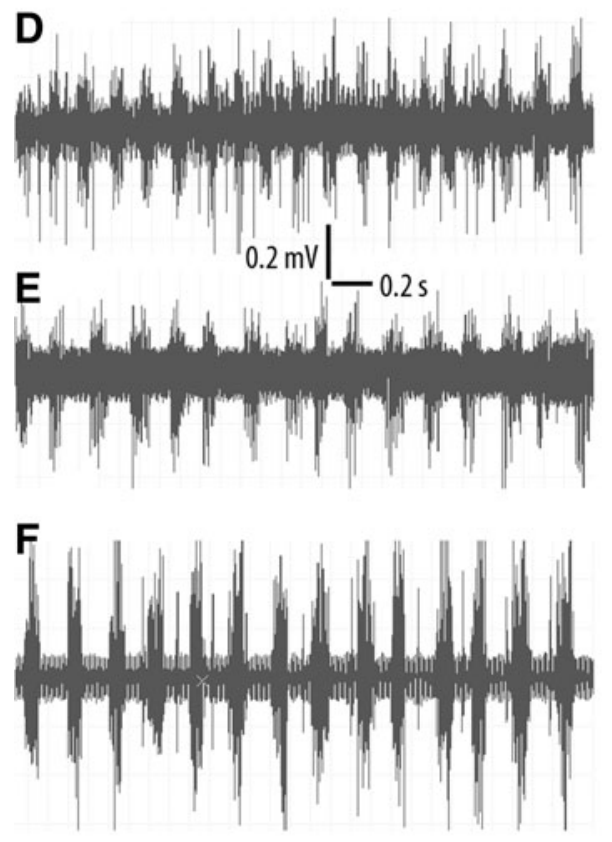
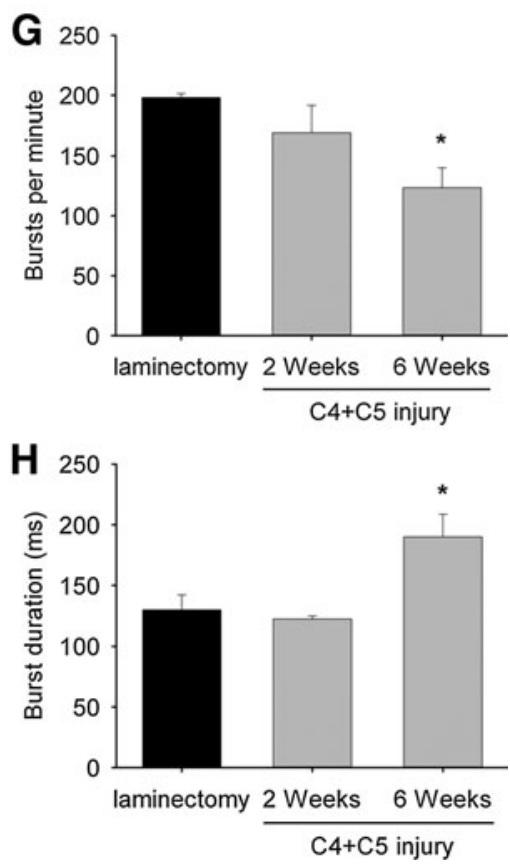

FIG. 1. Diaphragm electrophysiology following double $\mathrm{C} 4+\mathrm{C} 5$ unilateral cervical contusion spinal cord injury (SCI). Compared with laminectomy control animals (A), C4 + C5 injury mice (B) had reduced phrenic nerve compound muscle action potential (CMAP) amplitudes, a functional electrophysiological assay of phrenic nerve innervation of the diaphragm. Significant decreases in peak CMAP amplitudes were found in injured mice at 2 and 6 weeks post-injury (C). Spontaneous diaphragm electromyography (EMG) recordings showed similar motor unit potential activation amplitudes between laminectomy (D) and injured (E and $\mathbf{F})$ mice. Representative traces are from $\mathrm{C} 4+\mathrm{C} 5$ mice at 2 weeks post-injury $(\mathbf{E})$ or 6 weeks post-injury $(\mathbf{F})$. The time scale and amplitude for the traces are indicated. A slight decrease in inspiratory burst frequency was observed in injured animals, becoming significant at 6 weeks post-injury (G). Quantitative analysis of EMG signals also showed an increase in the duration of each discharge in injured animals at 6 weeks post-injury (H). Results are expressed as means $\pm \mathrm{SEM}$. ${ }^{*} \mathrm{C} 4+\mathrm{C} 5$ injury versus laminectomy $(p<0.05)$; $* * \mathrm{C} 4+\mathrm{C} 5$ injury versus laminectomy $(p<0.01) . n=8-10$ animals per group.

$190 \pm 19 \mathrm{~ms}, p<0.05$, in laminectomy and C4 + C5 injury, respectively) (Fig. 1H).

\section{Gray and white matter disruption}

We histologically characterized the rostral-caudal extension of lesion, as well as general morphology of gray and white matter regions, following $\mathrm{C} 4+\mathrm{C} 5$ contusion. Cresyl violet stained sections of cervical spinal cord from uninjured animals (Fig. 2E) and mice subjected to the injury paradigm (Fig. 2A-D) were analyzed for lesion area and volume at both 2 weeks and 6 weeks post-injury. In transverse spinal cord sections, the lesion epicenter encompassed the dorsolateral, lateral and ventrolateral funiculi and in a few cases reached the ventral funiculus at C5 level (Fig. 2B and C). Gray matter was mostly unspared at the lesion epicenter, as the general structure of dorsal and ventral horns was disrupted and filled in with hypercellularized fibrous tissue (Fig. 2F). At more rostral (C3) and caudal (C6) locations, lesioned tissue was mostly detected in white matter areas, especially in the lateral funiculus (Fig. 2A and D). At the injury epicenter at 2 weeks post-injury, lesion area reached a 
A

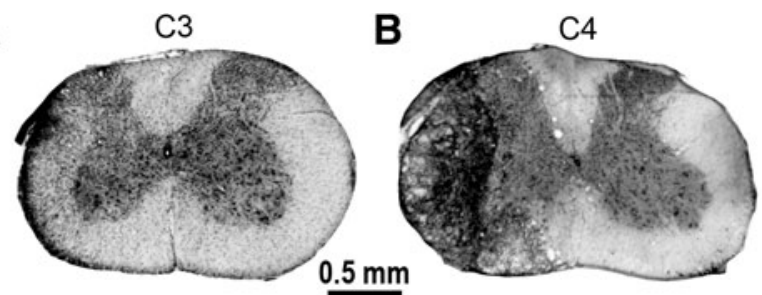

E

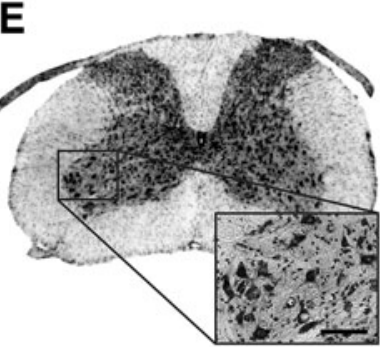

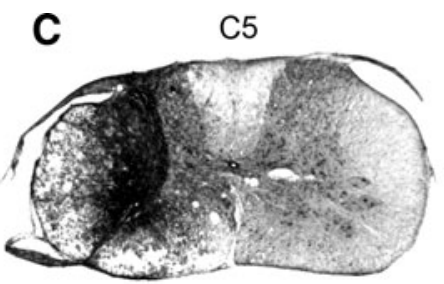
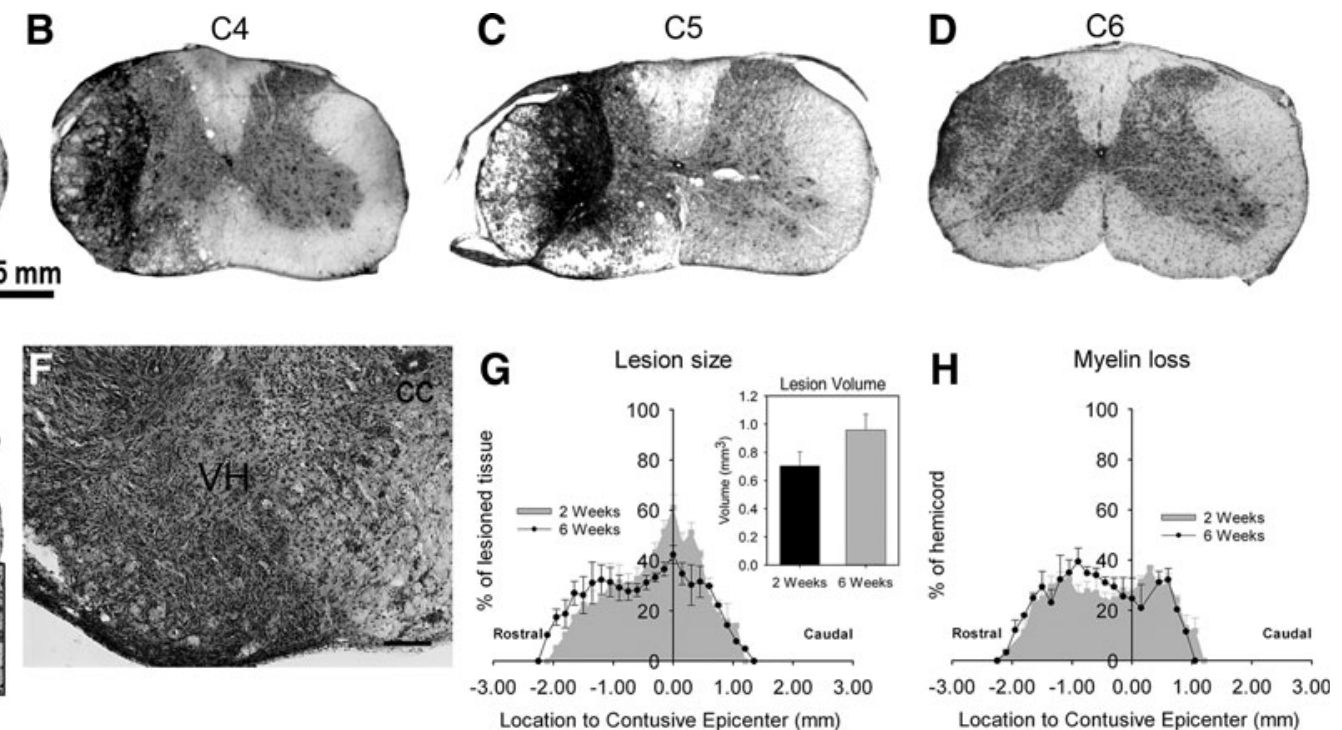

FIG. 2. Histological analyses of spinal cord following double $\mathrm{C} 4+\mathrm{C} 5$ unilateral cervical contusion spinal cord injury (SCI). Representative images illustrate the rostral boundary of the lesion at the $\mathrm{C} 3$ level (C3: A), the impact at C4 (C4: B), the lesion epicenter at C5 (C5: C), and the caudal edge at the C6 level (C6: D) at 2 weeks post-injury. The typical lesion in mice affected most of the gray matter at the epicenter. The lesion also disrupted several white matter regions, extending along the dorsolateral, lateral, and ventrolateral funiculi at $\mathrm{C} 4$ and reaching the ventral funiculus at $\mathrm{C} 5$. Evidence of white matter degeneration was observed in the lateral funiculus at C3 and C6 levels. The uninjured group did not show any pathological abnormalities following the laminectomy procedure (C4: E). Illustrations are representative of 5-7 independent mice in each group (scale bar: $0.5 \mathrm{~mm}$ ). Higher magnification of the lesion close to the epicenter is provided, showing the central canal (cc) and the ipsilateral ventral horn (VH) gray matter (scale bar: $100 \mu \mathrm{m})(\mathbf{F})$. Both gray and white matter regions were disrupted, hypercellularized and filled with dense fibrous tissue. Cresyl violet-stained motor neurons are seen in uninjured animals at all cervical regions (inset in E), whereas they are completely lost at the lesion epicenter (F). Quantitative lesion analyses did not show any significant difference in lesion extension or total lesion volume (G) between 2 and 6 weeks post-injury. Overall, the $\mathrm{C} 4+\mathrm{C} 5$ injury paradigm produced a lesion spreading over $3.5 \mathrm{~mm}$ in the rostral-caudal axis. Quantitative analyses of myelin loss did not show any extension of white matter damages beyond the lesion or any difference between 2 and 6 weeks post-injury (H). $n=5-7$ animals per group.

maximum of $61 \pm 5 \%$ of total ipsilateral hemi-cord area and was located at the C5 level. At 6 weeks post-injury, the lesion area at the epicenter encompassed $42 \pm 4 \%$ of total ipsilateral spinal cord area ( $p<0.05$ compared with 2 weeks) (Fig. 2G) and extended away from the epicenter in both rostral $(2.2 \mathrm{~mm})$ and caudal $(1.3 \mathrm{~mm})$ directions. This degree of rostral-caudal extension of the lesion was slightly smaller at 2 weeks post-injury. When lesion areas at regions extending throughout the cervical spinal cord were analyzed using Cavalieri's method, ${ }^{28}$ overall lesion volumes slightly increased over time between 2 and 6 weeks; however, this change was not statistically significant $\left(0.7 \pm 0.1 \mathrm{~mm}^{3}\right.$ and $1.0 \pm 0.1 \mathrm{~mm}^{3}$ respectively, $p>0.05$ ) (Fig. 2G). In addition, Eriochrome R stained sections were analyzed to quantify the area of unspared myelin in various white matter tracts. Myelin loss spread out in both rostral $(2.2 \mathrm{~mm})$ and caudal $(1.1 \mathrm{~mm})$ directions without any extension beyond the lesion previously detected by cresyl violet staining (Fig. 2H).

\section{Loss of total cervical motor neurons and phrenic motor neurons}

Cresyl violet stained sections of cervical spinal cord from mice that received a $\mathrm{C} 4+\mathrm{C} 5$ contusion SCI were further analyzed for total counts of large ventral horn motor neurons at 2 and 6 weeks post-injury (inset in Fig. 2E). Compared with laminectomy-only sham animals, mice receiving cervical contusion showed significant loss of ipsilateral ventral horn motor neurons at multiple distances from the lesion epicenter at both 2 and 6 weeks (Fig. 3A).
Interestingly, contralateral motor neuron numbers were significantly affected, but only at the level of the epicenter (Fig. 3B).

To specifically track the ipsilateral phrenic motor neuron pool, we injected cholera toxin $\beta$ subunit $(\mathrm{CT} \beta)$, a monosynaptic retrograde tracer, into the right hemidiaphragm. Motor neurons in the ventral horn that were labeled by retrograde transport of $\mathrm{CT} \beta$ from the hemidiaphragm were concentrated within a relatively compact group of cells forming a linear column throughout the ipsilateral ventral horn (Fig. 3D). As seen in both transverse (Fig. 3C) and sagittal (Fig. 3D) sections, labeled neurons were located in the gray matter and exhibited morphology consistent with motor neurons. These results revealed that the large $\mathrm{CT} \beta^{+}$neurons in the ventrolateral region of $\mathrm{C} 3-\mathrm{C} 5$ were phrenic motor neurons. Mice subjected to cervical contusion SCI (Fig. 3F) showed an obvious loss in the number of $\mathrm{CT} \beta^{+}$motor neurons compared with the laminectomy control group (Fig. 3E). Quantification demonstrated a significant decrease in $\mathrm{CT} \beta^{+}$cells at multiple distances around the lesion epicenter (Fig. 3G). The total number of phrenic motor neurons was estimated at $71 \pm 16$ motor neurons per animal after $\mathrm{C} 4+\mathrm{C} 5$ injury compared with $163 \pm 18$ in uninjured animals at 6 weeks $(p<0.01)$ (Fig. 3H).

\section{Phrenic nerve axonopathy}

As phrenic motor neuron loss was clearly established, we sought to characterize downstream effects on the phrenic respiratory pathway. Ipsilateral phrenic nerves were examined on Toluidine blue-stained, semi-thin sections. Numerous myelin ovoids and 
A Ipsilateral VH motor neurons
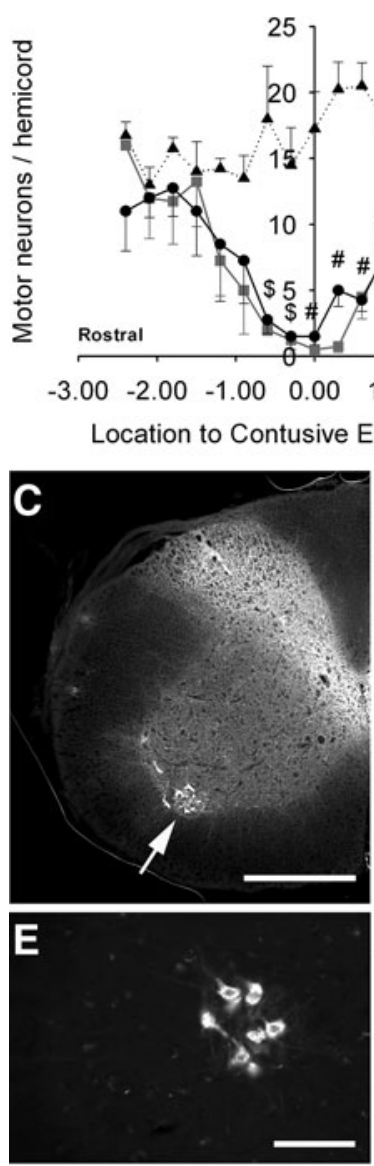

$\mathbf{F}$

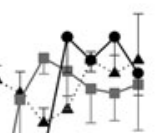

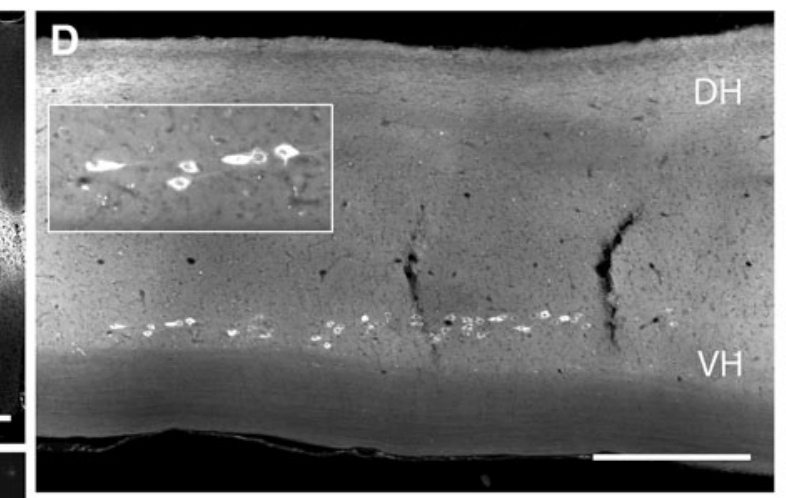

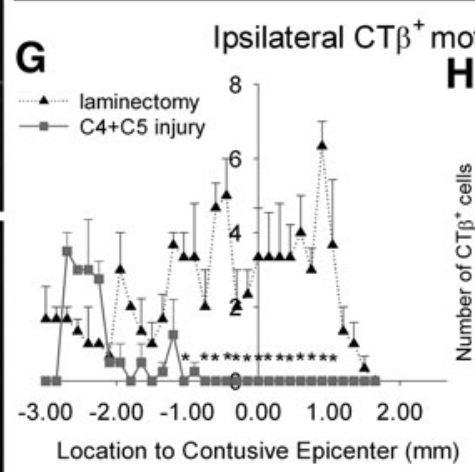

B Contralateral VH motor neurons

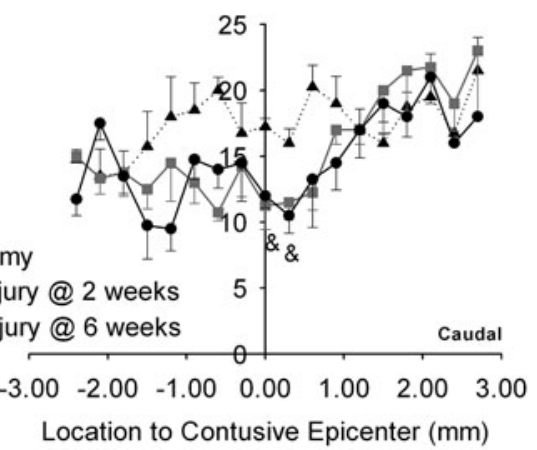

Location to Contusive Epicenter $(\mathrm{mm})$

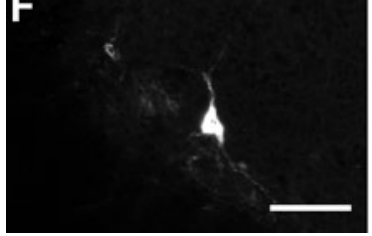

......... laminectomy

$\rightarrow-$ C4+C5 injury @ 2 weeks

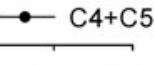

FIG. 3. Quantification of total cervical motor neuron and phrenic motor neuron loss. Large motor neurons were identified on cresyl violet stained sections and were manually counted in the ipsilateral or contralateral ventral horns (VH) following $\mathrm{C} 4+\mathrm{C} 5$ injury (A and B). Following injury, a significant loss of motor neurons over a length of $4 \mathrm{~mm}$ was observed surrounding the lesion epicenter in the ipsilateral VH (A). Contralateral hemi-spinal cord remained unaffected, except at two locations opposite the lesion epicenter, where the mean number of motor neurons was slightly reduced (B). There were no differences in motor neuron counts between 2 weeks (gray square) and 6 weeks (black dot) post-injury. The number of motor neurons in the cervical spinal cord from uninjured laminectomy-only mice is denoted by a black triangle. The phrenic motor neuron pool was visualized specifically in the cervical spinal cord using fluorescently labeled CT $\beta$ retrograde tracer injected in the intrapleural space of the ipsilateral hemi-diaphragm (C-H). Representative illustrations show transverse (C) and longitudinal (D) sections of the cervical spinal cord, revealing cholera toxin $\beta$ (CT $\beta$ )-labeled motor neurons (white arrow) in the VH. Inset in D: higher magnification image of CT $\beta^{+}$phrenic motor neurons forming a compact and linear column. Surrounding the lesion epicenter, an obvious difference in the number of CT $\beta$-labeled cells was observed between laminectomy (E) and injured (F) mice at 6 weeks post-injury. Bars represent $500 \mu \mathrm{m}$ in $\mathbf{C}$ and $\mathbf{D}$ and $100 \mu \mathrm{m}$ in $\mathbf{E}$ and $\mathbf{F}$. Quantification of $\mathrm{CT} \beta$-labeled cells at multiple distances from the lesion epicenter showed significant loss of phrenic motor neurons at multiple locations within the ipsilateral lesioned hemi-spinal cord at 6 weeks post-injury (G). Quantification of CT $\beta$-labeled cells shows a total loss of 56\% of the phrenic motor neuron pool in the ipsilateral lesioned hemi-spinal cord at 6 weeks post-injury $(\mathbf{H})$. Results are expressed as means \pm SEM. ${ }^{\$} \mathrm{C} 4+\mathrm{C} 5$ injury versus laminectomy $(p<0.05) ;{ }^{8} \mathrm{C} 4+\mathrm{C} 5$ injury versus laminectomy $(p<0.01) ;{ }^{\#} \mathrm{C} 4+\mathrm{C} 5$ injury versus laminectomy $(p<0.001) ; * \mathrm{C} 4+\mathrm{C} 5$ injury versus laminectomy at 6 weeks $(p<0.01) . n=5-7$ animals per group.

obvious axonal degeneration surrounded by large areas of fibrous tissue were detected following C4 + C5 injury (Fig. 4B), whereas the general morphology of myelinated fibers in uninjured animals appeared completely normal (Fig. 4A). Axon density in the phrenic nerve was significantly reduced in injured groups at 2 weeks and 6 weeks following injury $\left(8,899 \pm 686\right.$ and $8,253 \pm 1,079$ axons $/ \mathrm{mm}^{2}$ respectively) compared with the laminectomy group $(12,421 \pm$ 1,079 axons $/ \mathrm{mm}^{2}, p<0.05$ ) (Fig. 4C). Compared with laminectomy control, the mean cross-sectional area of individual axons was not significantly affected in the injury condition at either time point 

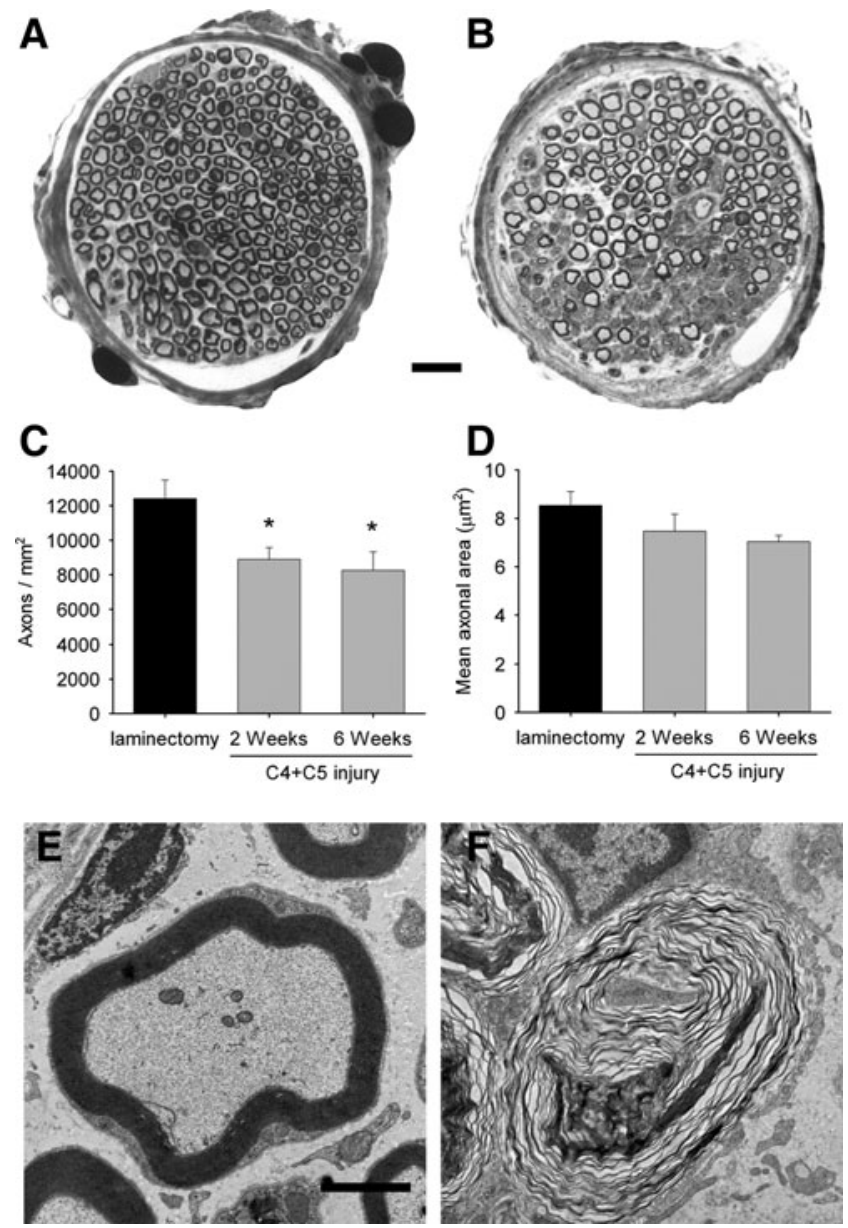

FIG. 4. Histological analysis of phrenic nerve. Phrenic nerves from $\mathrm{C} 4+\mathrm{C} 5$ injured (B) animals exhibited significant pathological changes such as degenerating fibers, proliferating Schwann cells, and endoneurial fibrosis, whereas abundant and compact myelinated fibers were found in the laminectomy control mice (A). The illustration in B is representative of 2 weeks post-injury, and a similar pattern of axonopathy was also observed at 6 weeks. Reduced density of myelinated axons was observed in ipsilateral phrenic nerves from injured mice at both time points compared with the laminectomy group (C). The average axonal area did not change significantly across conditions (D). Ultrastructural analysis of phrenic nerve sections from injured mice (F) shows degenerating axons surrounded by disrupted myelin sheath, compared with the normal morphology of axons in the laminectomy sham group (E). Bars represent $10 \mu \mathrm{m}$ in $\mathbf{A}$ and $\mathbf{B}$ and $1 \mu \mathrm{m}$ in $\mathbf{E}$ and $\mathbf{F}$. Results are expressed as means \pm SEM. $* \mathrm{C} 4+\mathrm{C} 5$ injury versus laminectomy $(p<0.05) ; n=5-7$ animals per group.

(Fig. 4D). Ultrastructural examination of ipsilateral phrenic nerves showed obvious axonal degeneration with axonal loss and myelin destruction suggestive of Wallerian degeneration (Fig. 4F), whereas the sham laminectomy group did not display any altered morphology (Fig. 4E).

\section{Normal diaphragm histology with NMJ abnormalities}

The histological appearance of hemidiaphragm muscles from laminectomy-only and injured mice was analyzed (Fig. 5A and B). Qualitatively, none of the groups showed gross morphological changes. Although hemidiaphragm muscles were extensively in- vestigated for total muscle thickness (Fig. 5C and D) and myofiber size (Fig. 5E) at five locations, no differences between uninjured and $\mathrm{C} 4+\mathrm{C} 5$ injured animals were detected.

To examine whether phrenic motor neuron loss and/or other injury-induced changes resulted in pathological alterations at the diaphragm NMJ, hemidiaphragm muscles were examined from laminectomy and injured animals 2 and 6 weeks post-injury. For this purpose, the hemidiaphragm was divided into three regions (ventral, central and dorsal) (Fig. 5F) for analysis, as the rostrocaudal axis of the phrenic motor neuron pool topographically maps onto the ventro-dorsal axis of the diaphragm. ${ }^{29}$

Ipsilateral hemidiaphragm from the sham group showed typical NMJ morphology (Fig. 5H), including thick pre-terminal axons and complete overlap of the pre-synaptic axon and post-synaptic acetylcholine receptors domains. In the injured mice, we observed severe NMJ disruption at the dorsal-most regions of the muscle (Fig. 5J), as well as milder effects at the central region, whereas the ventral part of the hemidiaphragm was mostly spared. To specifically characterize the types of changes occurring over time, NMJ morphologies were broken down into a number of phenotypic categories: intact (Fig. 5I), partially denervated (Fig. 5K), fully denervated (Fig. 5L), multiply innervated (Fig. 5M) and preterminal axon thinning (Fig. 5N). Uninjured animals had an average of $99.0 \%$ intact NMJs across all regions of the hemidiaphragm. Although quantification in injured animals demonstrated relative sparing of the ventral region $(92.3 \%$ of intact NMJs at 2 weeks), dorsal and central areas of the hemidiaphragm showed clear signs of synaptic abnormalities (Table 3). Specifically, only $39.0 \%$ of junctions $(p<0.01)$ in the central region and $6.9 \%$ of junctions $(p<0.001)$ in the dorsal region were completely intact (Fig. 5G). At 2 weeks post-injury, the dorsal area showed the greatest percentage of fully denervated junctions (refer to Table 3 for detailed analyses). At 6 weeks post-injury, signs of attempted reinnervation became obvious, as significant numbers of multiply reinnervated junctions were observed in central and dorsal regions $(9.5 \%, p<0.05$ and $13.9 \%, p<0.01$; compared with $0.0 \%$ in the laminectomy group) and the global number of denervated junctions decreased. Similarly, significant numbers of NMJs exhibited thin pre-terminal axon profiles $(9.3$ $\%, p<0.05$ and $12.3 \%, p<0.001$; compared with $0.6 \%$ in the laminectomy group) (Fig. $5 \mathrm{~N}$ ), which, along with multiply innervated NMJs (Fig. 5M), is characteristic of reinnervation after denervation. ${ }^{30}$ It is noteworthy that even at 6 weeks post-injury, the total numbers of intact junctions in the affected regions of the diaphragm were still significantly lower than in the laminectomy group $(p<0.05$ in the central region and $p<0.001$ in the dorsal region) (Fig. $5 \mathrm{G})$.

\section{Discussion}

We previously demonstrated that both single ( $\mathrm{C} 4$ only) and double $(\mathrm{C} 3+\mathrm{C} 4)$ unilateral contusion injuries at mid-cervical levels targeting the phrenic nucleus can be generated in the rat, resulting in long-lasting deficits in diaphragm function. ${ }^{29}$ The present study demonstrates that a similar contusion paradigm at both $\mathrm{C} 4$ and $\mathrm{C} 5$ levels can be generated in mice. To the best of our knowledge, the present work is the first to characterize a consistent experimental model of mid-cervical spinal contusion in mice that results in phrenic motor neuron degeneration and long-lasting unilateral deficits in diaphragm function. This work also addresses a gap in the knowledge regarding peripheral changes in respiratory circuitry following cervical contusion SCI. 
Because of the small size of the mouse, delivering a significant impact that produces quantifiable deficits in diaphragm function without a significant rate of mortality is a challenge following cervical contusion injuries. Because of potential effects on mortality, we chose to unilaterally target the phrenic motor neuron pool to preserve as much of the contralateral population as possible, while still significantly affecting ipsilateral hemidiaphragm function. The unilateral nature of the present injury likely allowed for a large proportion of animals to survive without the need for respiratory assistance. Nevertheless, a substantial number of animals still died ( 30\%) almost immediately following injury. The exact cause of death was not determined; however, the gasping observed in these mice during the initial minutes post-impact suggests that they died of respiratory failure. Previously developed contusion injuries at mid-cervical levels in rodents have also been associated with respiratory complications. ${ }^{6,23}$ Interestingly, the C5 impact was almost never associated with mortality in the present study, suggesting a greater susceptibility of overall ventilation to contusion injury at the $\mathrm{C} 4$ region. Preliminary data from our laboratory showed that generation of a single impact at the $\mathrm{C} 4$ level only was sufficient to obtain long-lasting deficits in ipsilateral hemi-diaphragm function (not shown); however, the double $\mathrm{C} 4+\mathrm{C} 5$ injury paradigm resulted in significantly greater functional compromise. Within 2 days post-injury, all mice resumed grooming and self-feeding behaviors and reliably displayed ipsilateral forelimb impairment. This clinical observation is in agreement with previous studies of cervical contusion in rats $^{20,26}$ and mice. ${ }^{6}$

Histological analysis of the spinal cord revealed features of spinal contusion typically observed in mice, including scar tissue, cell infiltration and absence of cystic cavity formation. The injury produced gray and white matter disruption at $\mathrm{C} 4$ and $\mathrm{C} 5$ levels that extended rostrally and caudally to C3 and C6, respectively, especially in white matter locations. Intriguingly, lesion size did not significantly increase between 2 and 6 weeks post-injury. Although edema and hemorrhage occurring during the acute phases of SCI resolve within days, secondary injury processes can cause additional cell death, tissue loss, and dysfunction for extended periods of time post-injury. ${ }^{31}$ It is likely that in this mouse model secondary degeneration is mostly complete within the first 2 weeks, as no further histological changes are detectable beyond this time window, at least when examined using conventional staining ap- proaches. Future work needs to examine possible ongoing pathological events, including axonal damage and glial cell death, at later time points with appropriate methods.

Similarly, we did not observe any significant differences in total motor neuron numbers between 2 and 6 weeks post-injury, suggesting no expansion of motor neuron cell loss beyond 2 weeks. Therefore, the therapeutic window for protecting phrenic motor neurons from secondary damage occurs between acute stages and 2 weeks post-injury; however, we do not provide any data regarding the temporal pattern of phrenic motor neuron loss occurring prior to 2 weeks post-injury. These types of analyses are crucial, as it is possible that the majority of motor neuron loss occurs during early stages post-injury (i.e., the first day or thereabouts).

Similar to previous studies in rodents, we localized the phrenic motor neuron pool mostly to levels $\mathrm{C} 3-\mathrm{C} 5,{ }^{18,25}$ with the highest concentration of labelled cells found at the $\mathrm{C} 4$ level. According to total counts of $\mathrm{CT} \beta^{+}$motor neurons, an $\sim 56 \%$ loss of ipsilateral phrenic motor neurons was observed following injury at 6 weeks. As we injected the CT $\beta$ tracer at 4 weeks post-injury and euthanized the animals at 6 weeks post-injury, the reduced numbers of CT $\beta$-labelled neurons could be explained in several ways in addition to phrenic motor neuron cell death, including impaired retrograde transport of the tracer caused by phrenic axon pathology and/ or severe denervation at the diaphragm NMJ. ${ }^{32,33}$ We have noted that the $\mathrm{CT} \beta$ fluorescent signal is gradually lost from phrenic motor neuron cell bodies at late time points post-injection. In order to avoid this issue, we chose to inject tracer into all mice close to the time of perfusion.

This injury paradigm also damaged white matter in the dorsolateral, lateral, and ventrolateral funiculi. Previous anterograde anatomical studies demonstrated that descending respiratory pathways that project to the phrenic nucleus are located in two regions of cervical spinal cord white matter: the lateral and ventromedial funiculi. ${ }^{34,35}$ In addition to loss of motor neuron cell bodies of the phrenic nucleus, double contusion injury targeting $\mathrm{C} 4$ and $\mathrm{C} 5$ levels also resulted in rostral extension of the lesion to the $\mathrm{C} 3$ segment. At this rostral level, damage specifically to lateral funiculus was observed, which might have resulted in additional effects on spontaneous diaphragm EMG recordings due to axonal damage and/or demyelination of the descending bulbospinal respiratory fibers.

For the first time, we have addressed the downstream pathological events occurring at the level of the phrenic nerve-

FIG. 5. Diaphragm histology and neuromuscular junction (NMJ) analysis. Hematoxylin and eosin staining did not show any observable pathological changes in ipsilateral hemidiaphragm muscle following C4 + C5 injury (B) compared with laminectomy-only mice (A). Bars represent $200 \mu \mathrm{m}$ in $\mathbf{A}$ and B. For quantitative analysis, three sections along the medial-lateral axis of the ipsilateral hemidiaphragm were analyzed per animal. On each section, the average of five thickness points equally distributed along the ventrodorsal axis was measured in a blind manner (C). Global muscle thickness (D) and myofiber size (E) did not show any changes at 2 or 6 weeks post-injury. Diaphragm NMJs were assessed across three different regions of the ipsilateral hemidiaphragm $(\mathbf{F})$ via labeling with rhodamine-alpha-bungarotoxin (red), SMI-312R (green) and SV2-s (green). Nearly all NMJs were completely intact in uninjured laminectomy mice (H and $\mathbf{I})$, characterized by: complete overlap of the pre-synaptic axon and pre-synaptic vesicles with post-synaptic acetylcholine receptors, no signs of multiple innervations, and absence of pre-synaptic axon thinning. Double C4 + C5 injury mice had strikingly fewer intact junctions at both time points (G) and displayed obvious areas of denervation in the hemidiaphragm $(\mathbf{J})$. To specifically examine the types of morphological changes occurring in the hemidiaphragm muscle, NMJ changes were broken down into a number of phenotypic categories: intact (I), partially denervated (K), fully denervated (L), and signs of reinnervation supported by the presence of multiply innervated NMJs $(\mathbf{M})$ and by thin preterminal axons $(\mathbf{N})$. Panels $\mathbf{J}$ and $\mathbf{L}$ provide an example of a fully denervated NMJ (\#1) and a partially denervated NMJ (\#2). Both types of denervated junctions were only found in C4 + C5 injured mice at both 2 and 6 weeks post-injury, mostly in the central and dorsal regions of the hemidiaphragm. Very rare instances of junctional pathologies were found in the laminectomy sham group. Details about the NMJ phenotypes are found in Table 3. Bars represent $20 \mu \mathrm{m}$ in $\mathbf{H}-\mathbf{N}$. Results are expressed as means \pm SEM. $* * \mathrm{C} 4+\mathrm{C} 5$ injury at 2 weeks versus laminectomy $(p<0.01)$; ***C4+C5 injury at 2 weeks

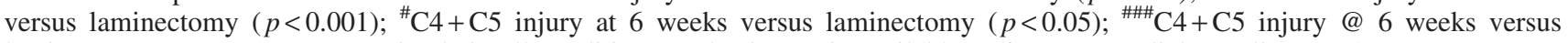
laminectomy $(p<0.001) . n=3$ animals in all conditions. Color image is available online at www.liebertonline.com/neu. 

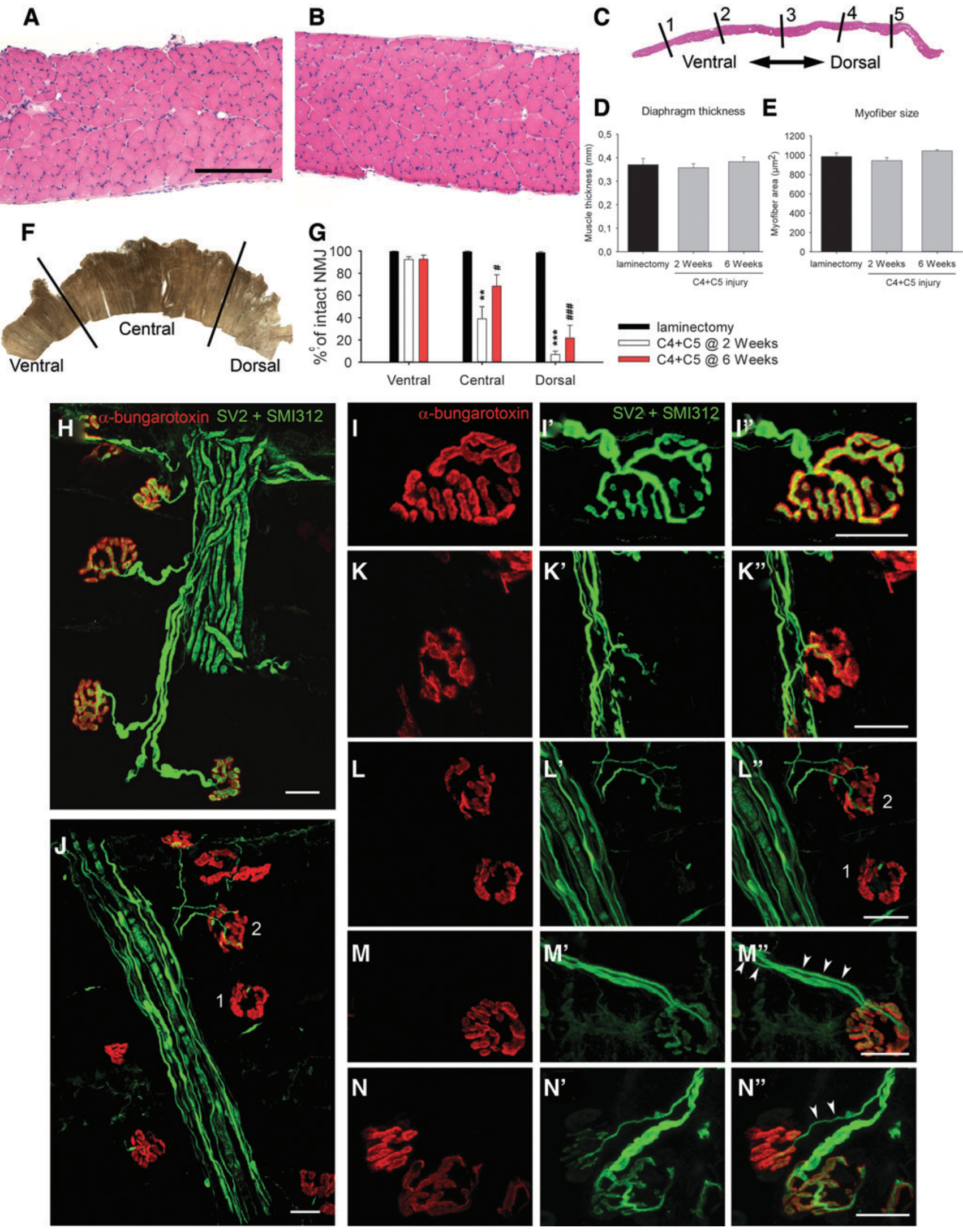
Table 3. Neuromuscular Junction (NMJ) Analysis

\begin{tabular}{|c|c|c|c|}
\hline & Laminectomy & $C 4+C 5$ at 2 weeks & $C 4+C 5$ at 6 weeks \\
\hline \multicolumn{4}{|l|}{ Ventral } \\
\hline Intact & $99.4 \pm 2.3$ & $92.3 \pm 2.5$ & $92.5 \pm 3.8$ \\
\hline Fully denervated & $0.0 \pm 0.0$ & $0.7 \pm 0.7$ & $1.9 \pm 1.3$ \\
\hline Partially denervated & $0.0 \pm 0.0$ & $1.0 \pm 0.6$ & $2.4 \pm 1.2$ \\
\hline Multiply innervated & $0.0 \pm 0.0$ & $2.4 \pm 1.5$ & $1.2 \pm 0.8$ \\
\hline Thin axons & $0.6 \pm 0.3$ & $3.6 \pm 1.9$ & $2.0 \pm 1.0$ \\
\hline \multicolumn{4}{|l|}{ Central } \\
\hline Intact & $99.3 \pm 0.5$ & $39.0 \pm 11.0 * *$ & $68.4 \pm 10.1 \#$ \\
\hline Fully denervated & $0.0 \pm 0.0$ & $18.5 \pm 7.3 *$ & $2.5 \pm 0.3$ \\
\hline Partially denervated & $0.1 \pm 0.1$ & $28.4 \pm 5.0 * *$ & $10.3 \pm 4.4$ \\
\hline Multiply innervated & $0.0 \pm 0.0$ & $6.0 \pm 1.3$ & $9.5 \pm 3.3^{\#}$ \\
\hline Thin axons & $0.6 \pm 0.4$ & $8.0 \pm 0.4$ & $9.3 \pm 3.1{ }^{\#}$ \\
\hline \multicolumn{4}{|l|}{ Dorsal } \\
\hline Intact & $98.4 \pm 1.0$ & $6.9 \pm 3.0 * * *$ & $21.7 \pm 11.4$ \#\#\# \\
\hline Fully denervated & $0.0 \pm 0.0$ & $55.2 \pm 7.8 * * *$ & $21.8 \pm 1.6^{\#, \S \S}$ \\
\hline Partially denervated & $1.0 \pm 0.5$ & $25.8 \pm 6.4$ & $30.3 \pm 8.5^{\#}$ \\
\hline Multiply innervated & $0.0 \pm 0.0$ & $5.9 \pm 1.7$ & $13.9 \pm 2.0$ \\
\hline Thin axons & $0.6 \pm 0.6$ & $6.2 \pm 0.7 * *$ & $12.3 \pm 1.0$ \#\#\#, $\S \S$ \\
\hline
\end{tabular}

NMJs were analysed in three regions of the ipsilateral hemidiaphragm and assigned to different morphological categories (intact, fully denervated, partially denervated, multiply innervated, preterminal thin axon). Whereas the ventral region was relatively spared following double $\mathrm{C} 4+\mathrm{C} 5$ injury (92.3\%), central and dorsal areas displayed significant loss of intact NMJs (39\% and 6.9\%, respectively) as early as 2 weeks post-injury. Abnormal NMJs morpholgies were further classified as either fully and partially denervated. A greater percentage of fully denervated NMJs was observed in the dorsal region $(55.2 \%)$ compared with the central region $(18.5 \%)$. The dorsal region was the most affected area of denervation across the hemidiaphragm. Significant morphological recovery was observed at 6 weeks post-injury in both central and dorsal areas, as detected by the presence of multiply innervated NMJs and preterminal thin axon, both signs of re-innervation following denervation.

*Laminectomy versus 2 weeks $(p<0.05)$; **Laminectomy versus 2 weeks $(p<0.01)$; ***Laminectomy versus 2 weeks $(p<0.001)$; ${ }^{*}$ Laminectomy

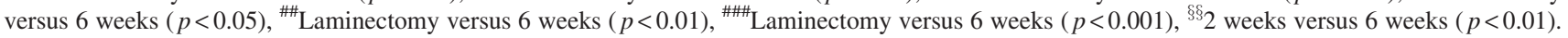
$n=3$ animals per group.

diaphragm circuit in a murine model of mid-cervical contusion SCI. Phrenic nerve histology demonstrated significant changes in nerve morphology such as signs of Wallerian degeneration, fibrous scar tissue, and reduced axon numbers, which was the likely cause of subsequent diaphragm NMJ abnormalities. We cannot rule out whether axonal loss in the phrenic nerve and all observed downstream events might be caused by direct phrenic axon damage at the level of the ventral roots during the impact. Our in-depth diaphragm NMJ analysis further demonstrates the phrenic axonal degeneration process, as many motor endplates were found to be denervated as early as 2 weeks post-injury. Interestingly, the pattern of diaphragm denervation followed a specific topography, as only the central and dorsal regions of the hemidiaphragm were affected. This observation is consistent with the preferential innervation of the dorsal region of the diaphragm by phrenic motor neurons located at the $\mathrm{C} 5$ level of the spinal cord. ${ }^{29}$ When we generated a double injury at $\mathrm{C} 3$ and $\mathrm{C} 4$ levels in the rat, denervation targeted mostly the ventral portion of the ipsilateral hemidiaphragm and caused significant muscular atrophy. ${ }^{26}$ This topographical relationship might be relevant for directing therapy to preserve and/or modulate diaphragm NMJ connectivity or to target a specific cervical level in the spinal cord following SCI. Although severe and long-lasting muscle denervation is known to cause muscular atrophy, we were unable to detect any changes either at the level of global diaphragm thickness or single myofiber size. This might be explained by the rapid and numerous reinnervation events observed as early as 6 weeks postinjury, possibly compensating for denervation caused by phrenic motor neuron loss. However, these compensatory reinnervation mechanisms were not functionally sufficient, as CMAP amplitudes were chronically reduced at 6 weeks post-injury to the same extent as 2 weeks post-injury.
Overall, the in vivo electrophysiological findings support the notion that the entire phrenic motor neuron-diaphragm respiratory pathway is chronically affected in this cervical contusion SCI paradigm. By measuring spontaneous EMG recordings under eupnea conditions in the present study, we found several alterations at 6 weeks post-injury, including decreased burst frequency and increased burst duration. Burst frequency and duration are rhythmic diaphragmatic parameters thought to be primarily regulated by descending bulbospinal input to the phrenic nucleus. ${ }^{19,36-38}$ Changes in diaphragm EMGs observed following contusion injury likely depend both on the location and degree of spared phrenic motor neurons ( $\sim 44 \%$ in the present study, most of them rostral to the C4 level) and on sparing/plasticity of descending bulbospinal respiratory input. In particular, we show that the ventromedial funiculus of the ipsi-lesioned spinal cord remained visually undamaged (i.e., intact, myelinated axons) rostral to $\mathrm{C} 4$. This sparing differs from the extensive damage observed in the lateral funiculus, the location of the primary descending respiratory tract. In uninjured animals, the ventromedial tract appears to act as a minor contributor to phrenic motor neuron activity. Following partial lateral C2 hemisection, long-term ipsilateral phrenic activity depends on this alternative descending respiratory pathway, independently of the lateral crossed phrenic pathway. ${ }^{39-41}$ In our model, the spared ventromedial region might mediate respiratory plasticity leading to functional network reorganization and consequent long-term modification of diaphragm EMG activity. Although not explored in the current study, these long-term diaphragm changes could also be caused by mechanisms such as morphological plasticity and changes in intrinsic excitability and inhibitory synaptic input to spared ipsilateral phrenic motor neurons, as well as plasticity in respiratory interneuron populations of the cervical spinal cord. ${ }^{41-43}$ 


\section{Conclusion}

In summary, we have described in the present report a model of mid-cervical spinal cord contusion in the adult mouse that induces unilateral diaphragm deficits within 2 weeks post-injury and that persists for at least 6 weeks. We have demonstrated that phrenic nerve conduction studies and diaphragm EMG recordings can serve as reliable, long-term functional outcome measures of respiratory dysfunction in models of cervical SCI. We speculate that degeneration of the phrenic motor nucleus ipsilateral to hemicontusion primarily accounts for the chronic nature of diaphragm deficits. Despite the plasticity described in respiratory pathways following various types of cervical SCI, the level of motor neuron degeneration described in this study still resulted in persistent diaphragm dysfunction. From a translational perspective, this would represent a compelling example of gray matter repair being an important option for SCI therapy.

With no need for respiratory assistance, our model is functionally and histologically reproducible and clinically relevant to a significant percentage of human SCI cases. Several contusion models at mid-cervical levels $(\mathrm{C} 3, \mathrm{C} 4, \mathrm{C} 5)$ that show chronic abnormalities in respiratory parameters have been developed in the rat. $^{22-25}$ The extension of a contusion injury model to the mouse provides opportunities for manipulating genes of interest and for evaluating their role in relevant outcome measures affected by cervical spinal contusion such as forelimb motor function, respiratory function, and neuropathic pain.

\section{Acknowledgments}

This work was supported by the Université Libre de Bruxelles (Bureau des Relations Internationales et de la Coopération, grant BRIC-11/092 to C.N.), the Craig Nielsen Foundation (grant \#190140 to A.C.L.), and the Paralyzed Veterans of America (grant \#160837 to A.C.L.). We are grateful to Michele Authelet and Ricardo Nieves for technical assistance. We thank Dr. Warren Alilain for helpful discussion regarding EMG data. We thank Aileen Anderson and Rebecca Nishi (funded by the Christopher and Dana Reeve Foundation SCI Core Facility) for valuable advice in setting up the cervical contusion model in mice.

\section{Author Disclosure Statement}

No competing financial interests exist.

\section{References}

1. Strakowski, J.A., Pease, W.S., and Johnson, E.W. (2007). Phrenic nerve stimulation in the evaluation of ventilator-dependent individuals with C4- and C5-level spinal cord injury. Am. J. Phys. Med. Rehabil. 86, 153-157.

2. Sharma, H., Alilain, W.J., Sadhu, A., and Silver, J. (2011). Treatments to restore respiratory function after spinal cord injury and their implications for regeneration, plasticity and adaptation. Exp. Neurol. 25, $18-25$.

3. Ma, M., Basso, D.M., Walters, P., Stokes, B.T., and Jakeman, L.B. (2001). Behavioral and histological outcomes following graded spinal cord contusion injury in the C57B1/6 mouse. Exp. Neurol. 169, 239254.

4. Myers, S.A., DeVries, W.H., Andres, K.R., Gruenthal, M.J., Benton, R.L., Hoying, J.B., Hagg, T., and Whittemore, S.R. (2011). CD47 knockout mice exhibit improved recovery from spinal cord injury. Neurobiol. Dis. 42, 21-34.

5. Okada, S., Nakamura, M., Katoh, H., Miyao, T., Shimazaki, T., Ishii, K., Yamane, J., Yoshimura, A., Iwamoto, Y., Toyama, Y., and Okano, H. (2006). Conditional ablation of Stat 3 or Socs3 discloses a dual role for reactive astrocytes after spinal cord injury. Nat. Med. 12, 829-834.
6. Aguilar, R.M., and Steward, O. (2010). A bilateral cervical contusion injury model in mice, assessment of gripping strength as a measure of forelimb motor function. Exp. Neurol. 221, 38-53.

7. Lepore, A.C., O'Donnell, J., Kim, A.S., Yang, E.J., Tuteja, A., Haidet-Phillips, A., O'Banion, C.P., and Maragakis, N.J. (2011). Reduction in expression of the astrocyte glutamate transporter, GLT1, worsens functional and histological outcomes following traumatic spinal cord injury. Glia 59, 1996-2005.

8. Marques, S.A., Garcez, V.F., Del Bel, E.A., and Martinez, A.M. (2009). A simple, inexpensive and easily reproducible model of spinal cord injury in mice: morphological and functional assessment. J. Neurosci. Methods 177, 183-193.

9. Anderson, K.D., Abdul, M., and Steward, O. (2004). Quantitative assessment of deficits and recovery of forelimb motor function after cervical spinal cord injury in mice. Exp. Neurol. 190, 184-191.

10. Carter, L.M., McMahon, S.B., and Bradbury, E.J. (2011). Delayed treatment with chondroitinase $\mathrm{ABC}$ reverses chronic atrophy of rubrospinal neurons following spinal cord injury. Exp. Neurol. 228, 149-156.

11. Duffy, P., Schmandke, A., Sigworth, J., Narumiya, S., Cafferty, W.B., and Strittmatter, S.M. (2009). Rho-associated kinase II (ROCKII) limits axonal growth after trauma within the adult mouse spinal cord. J. Neurosci. 29, 15,266-15,276.

12. Harel, N.Y., Song, K.H., Tang, X., and Strittmatter, S.M. (2010). Nogo receptor deletion and multimodal exercise improve distinct aspects of recovery in cervical spinal cord injury. J. Neurotrauma 27, 2055-2066.

13. Minor, K.H., Akison, L.K., Goshgarian, H.G., and Seeds, N.W. (2006). Spinal cord injury-induced plasticity in the mouse-the crossed phrenic phenomenon. Exp. Neurol. 200, 486-495.

14. Goshgarian, H.G. (2003). The crossed phrenic phenomenon: a model for plasticity in the respiratory pathways following spinal cord injury. J. Appl. Physiol. 94, 795-810.

15. Baussart, B., Stamegna, J.C., Polentes, J., Tadie, M., and Gauthier, P. (2006). A new model of upper cervical spinal contusion inducing a persistent unilateral diaphragmatic deficit in the adult rat. Neurobiol. Dis. $22,562-574$

16. Norenberg, M.D., Smith, J., and Marcillo, A. (2004). The pathology of human spinal cord injury: defining the problems. J. Neurotrauma 21, 429-440.

17. Jakeman, L.B., Chen, Y., Lucin, K.M., and McTigue, D.M. (2006). Mice lacking L1 cell adhesion molecule have deficits in locomotion and exhibit enhanced corticospinal tract sprouting following mild contusion injury to the spinal cord. Eur. J. Neurosci. 23, 1997-2011.

18. Qiu, K., Lane, M.A., Lee, K.Z., Reier, P.J., and Fuller, D.D. (2010). The phrenic motor nucleus in the adult mouse. Exp. Neurol. 226, 254 258.

19. Alilain, W.J., Li, X., Horn, K.P., Dhingra, R., Dick, T.E., Herlitze, S., and Silver, J. (2008). Light-induced rescue of breathing after spinal cord injury. J. Neurosci. 28, 11,862-11,870.

20. Choi, H., Liao, W.L., Newton, K.M., Onario, R.C., King, A.M., Desilets, F.C., Woodard, E.J., Eichler, M.E., Frontera, W.R., Sabharwal, S., and Teng, Y.D. (2005). Respiratory abnormalities resulting from midcervical spinal cord injury and their reversal by serotonin $1 \mathrm{~A}$ agonists in conscious rats. J. Neurosci. 25, 4550-4559.

21. el-Bohy, A.A., Schrimsher, G.W., Reier, P.J., and Goshgarian, H.G. (1998). Quantitative assessment of respiratory function following contusion injury of the cervical spinal cord. Exp. Neurol. 150, 143152.

22. Golder, F.J., Fuller, D.D., Lovett-Barr, M.R., Vinit, S., Resnick, D.K., and Mitchell, G.S. (2011). Breathing patterns after mid-cervical spinal contusion in rats. Exp. Neurol. 231, 97-103.

23. Lane, M.A., Lee, K.Z., Salazar, K., O'Steen, B.E., Bloom, D.C., Fuller, D.D., and Reier, P.J.. (2011). Respiratory function following bilateral mid-cervical contusion injury in the adult rat. Exp. Neurol. 235, 297-210.

24. Lepore, A.C. (2011). Intraspinal cell transplantation for targeting cervical ventral horn in amyotrophic lateral sclerosis and traumatic spinal cord injury. J Vis Exp. 18, 3069.

25. Mantilla, C.B., Zhan, W.Z., and Sieck, G.C. (2009). Retrograde labeling of phrenic motoneurons by intrapleural injection. J. Neurosci. Methods 182, 244-249.

26. Nicaise, C., Hala, T.J., Frank, D.M., Parker, J.L., Authelet, M., Leroy, K., Brion, J.P., Wright, M.C., and Lepore, A.C. (2012). Phrenic motor neuron degeneration compromises phrenic axonal circuitry and dia- 
phragm activity in a unilateral cervical contusion model of spinal cord injury. Exp. Neurol. 235, 539-552.

27. Wright, M.C., and Son, Y.J. (2007). Ciliary neurotrophic factor is not required for terminal sprouting and compensatory reinnervation of neuromuscular synapses, re-evaluation of CNTF null mice. Exp. Neurol. 205, 437-448.

28. Cavalieri, B. (1966). Geometria Degli Indivisibili (original edition, Bologna 1635). Unione Tipografico-Editrice Torinese: Torino.

29. Laskowski, M.B., and Sanes, J.R. (1987). Topographic mapping of motor pools onto skeletal muscles. J. Neurosci. 7, 252-260.

30. Son, Y.J., and Thompson, W.J. (1995). Schwann cell processes guide regeneration of peripheral axons. Neuron 14, 125-132.

31. Oyinbo, C.A. (2011). Secondary injury mechanisms in traumatic spinal cord injury: a nugget of this multiply cascade. Acta Neurobiol. Exp. (Wars). 71, 281-299.

32. Dumas, M., Schwab, M.E., and Thoenen, H. (1979). Retrograde axonal transport of specific macromolecules as a tool for characterizing nerve terminal membranes. J. Neurobiol. 10, 179-197.

33. Oldfors, A. (1986). Cholera toxin B-subunit incorporation into synaptic vesicles of the neuromuscular junction of the rat. Experientia 42 , 415-417.

34. Feldman, J.L., Loewy, A.D., and Speck, D.F. (1985). Projections from the ventral respiratory group to phrenic and intercostal motoneurons in cat: an autoradiographic study. J. Neurosci. 5, 1993-2000.

35. Lipski, J., Zhang, X., Kruszewska, B., and Kanjhan, R. (1994). Morphological study of long axonal projections of ventral medullary inspiratory neurons in the rat. Brain Res. 640, 171-184.

36. Bellingham, M.C. (1999). Synaptic inhibition of cat phrenic motoneurons by internal intercostal nerve stimulation. J. Neurophysiol. 82, 1224-1232.

37. Feldman, J.L., and Del Negro, C.A. (2006). Looking for inspiration: new perspectives on respiratory rhythm. Nat. Rev. Neurosci. 7, 232-242.
38. Smith, J.C., Ellenberger, H.H., Ballanyi, K., Richter, D.W., and Feldman, J.L. (1991). Pre-Botzinger complex: a brainstem region that may generate respiratory rhythm in mammals. Science 254, 726-729.

39. Vinit, S., Darlot, F., Stamegna, J.C., Sanchez, P., Gauthier, P., and Kastner, A. (2008). Long-term reorganization of respiratory pathways after partial cervical spinal cord injury. Eur. J. Neurosci. 27, 897-908.

40. Vinit, S., Gauthier, P., Stamegna, J.C., and Kastner, A. (2006). High cervical lateral spinal cord injury results in long-term ipsilateral hemidiaphragm paralysis. J. Neurotrauma 23, 1137-1146.

41. Vinit, S., and Kastner, A. (2009). Descending bulbospinal pathways and recovery of respiratory motor function following spinal cord injury. Respir. Physiol. Neurobiol. 169, 115-122.

42. Lane, M.A., Fuller, D.D., White, T.E., and Reier, P.J. (2008). Respiratory neuroplasticity and cervical spinal cord injury: translational perspectives. Trends Neurosci. 31, 538-547.

43. Lane, M.A., Lee, K.Z., Fuller, D.D., and Reier, P.J. (2009). Spinal circuitry and respiratory recovery following spinal cord injury. Respir. Physiol. Neurobiol. 169, 123-132.

\author{
Address correspondence to: \\ Angelo C. Lepore, Ph.D. \\ Department of Neuroscience \\ Farber Institute for Neurosciences \\ Thomas Jefferson University Medical College \\ 900 Walnut Street, JHN 469 \\ Philadelphia, PA 19107
}

E-mail: Angelo.Lepore@jefferson.edu 